\title{
Adjuvant radiation therapy after immediate implant-based breast reconstruction
}

\author{
Joanna Socha ${ }^{1,2}$ \\ 'Department of Radiotherapy, Military Institute of Medicine, Warsaw, Poland \\ ${ }^{2}$ Department of Radiotherapy, Regional Oncology Centre, Czestochowa, Poland
}

The role of radiotherapy in the postmastectomy setting with substantial lymph node burden or locally advanced disease has been well described. In the last decade, the indications for postmastectomy radiotherapy (PMRT) have expanded in light of a measurable disease-free survival benefit, even in T1-2N1-patient subgroup. Concurrently, immediate breast reconstruction (IBR) rates after mastectomy are rapidly increasing. Optimal integration of IBR and PMRT is challenging, as PMRT has a known deleterious effect on reconstruction outcomes and IBR has been reported to pose challenges to PMRT delivery. Implant-based reconstruction is the most common type of IBR performed nowadays. This article reviews the current problems regarding integration of the implant-based IBR with optimum radiation delivery and discusses the advantages and disadvantages of each reconstruction method with PMRT.

NOWOTWORY J Oncol 2020; 70, 1: 20-25

Key words: adjuvant radiotherapy, alloplastic reconstruction, immediate breast reconstruction, implant-based reconstruction, postmastectomy radiotherapy

\section{Introduction}

Immediate breast reconstruction (IBR) rates have continued to increase over time, concurrently with expanded indications for postmastectomy radiation therapy (PMRT) resulting from evidence that PMRT reduces recurrences and breast cancer mortality not only in patients with substantial lymph node burden or locally advanced disease, but also in pT1-2N1-patient subgroup [1, 2]. Although surgeons used to anticipate receipt of PMRT to guide decision-making regarding recommendations for IBR, nowadays a tendency for women with more advanced tumors to be less likely offered IBR due to their overall poorer prognosis and very high likelihood of receiving PMRT is gradually decreasing [3]. Optimal integration of IBR and PMRT is challenging, as PMRT has a known deleterious effect on reconstruction outcomes [4-6] and IBR has been reported to pose challenges to PMRT delivery [7]. The implant-based IBR (IB-IBR) is usually preferable in the majority of patients with breast cancer facing PMRT due to its preservation of autologous tissue for salvage and often acceptable outcomes, whereas most guidelines do not routinely recommend autologous reconstruction in patients who will definitely need PMRT [8, 9]. In current practice, reconstruction with tissue expander (TE) followed by PMRT and subsequent permanent reconstruction with prosthesis is prevalent [10].

The aim of this article is to review the current problems regarding integration of IB-IBR with optimum radiation delivery and to discuss the advantages and disadvantages of each reconstruction method with PMRT. Several questions will be addressed, such as oncological safety, cosmetic outcomes, and some technical radiotherapy issues, like target volume definitions depending on the reconstruction methods and disease stage, a problem of administering a boost and of using bolus material, the volume of fluid within the TE - i.e. deflation or inflation before PMRT, and the impact of an internal magnetic metallic port within TE on radiotherapy dose distribution. 


\section{PMRT in patients after IB-IBR - is oncological safety compromised?}

IBR improves quality of life and self-perceived body image [11]. However, a concern remains that the procedure may have an impact on disease control, resulting from the risk of delaying PMRT due to surgical complications and from the influence of IBR on the optimization of PMRT, compromising dose coverage of the clinical target volume (CTV), i.e. of the volume of tissues that contains subclinical malignant disease at a certain probability level, and thus has to be treated adequately. There are no data from prospective randomized trials on the oncological safety of IBR followed by PMRT. Two meta-analyses have reported that local recurrence rates [12], overall survival and disease-free survival [13] did not differ between patients with or without IBR. However, not more than 30\% of the patients included in these meta-analyses were treated with PMRT, detailed data about RT were missing in several included studies, most of the patients included had the early stage of disease (clinical stage I-II) and patients with IB-IBR constituted the minority of patients in these meta-analyses.

Results of a matched control study where the population consisted of 128 IB-IBR patients (all with retropectoral implants, one third irradiated with TE) and 252 controls without IBR, aiming to evaluate the CTV dose coverage and to investigate the safety of IB-IBR in terms of recurrence and survival compared to patients without an implant, showed that PMRT after IB-IBR lead to minor under-dosage of the CTV. However, recurrence and survival rates were equally distributed among patients with IB-IBR and controls, indicating that the overall treatment protocol is safe [14]. Again, patients with locally advanced breast cancer (LABC), i.e. pT3 disease, constituted less than $10 \%$ of the whole cohort, and patients who received neoadjuvant chemotherapy constituted only $24.2 \%$ of the IB-IBR group.

In a population-based propensity score matched analysis comparing the survival outcomes in LABC patients (pT1-4N2$3 \mathrm{MO}$ ) receiving PMRT with and without IBR, that included 1732 patients from the Surveillance, Epidemiology, and End Results (SEER) database, there was comparable breast cance r specific survival and overall survival between patients who received IBR or mastectomy alone followed by PMRT. In this study, 36.8\% of patients received autologous IBR, 36.3\% received IB-IBR, and $26.8 \%$ had reconstruction that was not otherwise specified or combined with tissue and implant reconstruction. PT1-T2 patients constituted $70 \%$ of the matched cohort [15].

With the increasing rates of prepectoral reconstructions being performed, often without the use of acellular dermal matrix (ADM) due to reimbursement policy in Poland, the problem emerged regarding the positional uncertainty of the target during PMRT, which is much higher in case of prepectoral TEs without ADM, due to higher range of both their inter- and intrafraction motion. It seems that anatomical position of prepectoral TEs without ADM is far less stable than retropectoral TE/implants and prepectoral implants with ADM. This may cause additional delays in PMRT as well as unplanned treatment breaks resulting from the need for re-planning of these patients whose initial target positioning cannot be reproduced during PMRT (Fig. 1). Such delays may have a negative impact on the oncological outcomes, as prolongation of the overall treatment time was confirmed as a cause of treatment failure in early breast cancer patients [16].

In conclusion, IB-IBR followed by PMRT seems safe for early stage patients (pT1-2N1), for whom the minor under-dosage of the CTV may be acceptable. However, caution should be paid when offering IB-IBR to the patients with locally advanced breast cancer, because data on the oncological safety are scarce, and there is virtually no data on patients with pT4-disease treated with IB-IBR followed by PMRT.

\section{Cosmetic outcomes}

Surgical techniques for IB-IBR continue to develop with the aim of improving cosmetic outcomes. However, in patients undergoing PMRT, adverse events must be considered, including the risk of reconstruction failure or major complications, such as capsular contracture or implant exposure $[17,18]$.

Reconstruction failure rates, being consistently reported at the level of about 20\%, are clinically significant when considering IB-IBR in the setting of PMRT $[4,18]$. Capsular contracture is a well-recognized complication of IB-IBR, which can occur in the absence of PMRT, because all breast implants become surrounded by scar tissue or fibrosis, and in some cases, excessive fibrosis re-

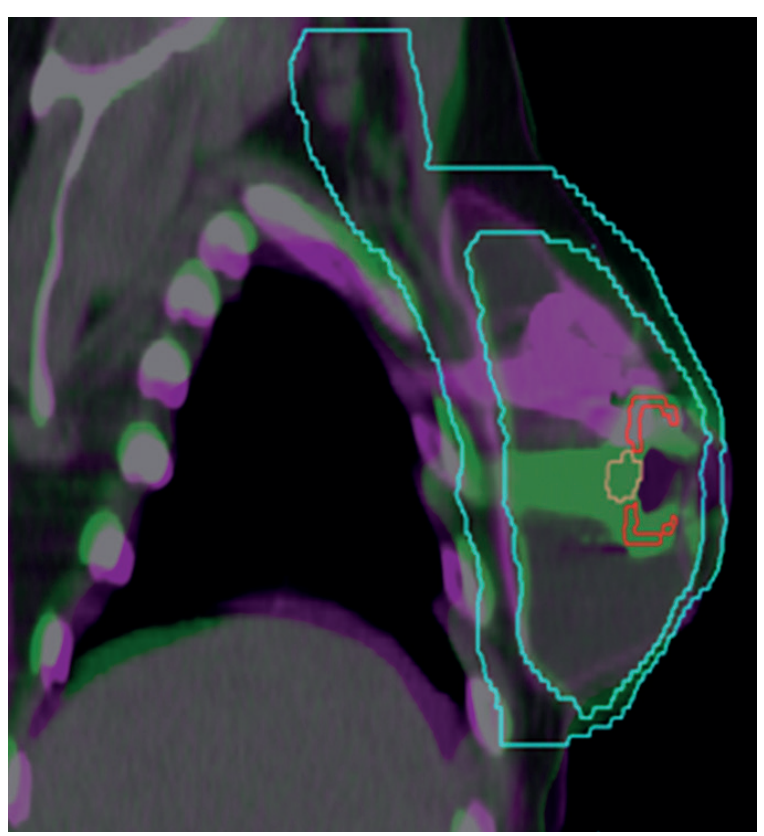

Figure 1. Anatomical position of prepectoral tissue expanders without acellular dermal matrix is not stable. Initial target positioning may be unreproducible, which results with additional delays or unplanned breaks in post-mastectomy radiation therapy due to the need for replanning. Localization computed tomography image (pink) fused with the reference image (green). Clinical target volume - blue; magnetic metallic port - red and orange. The lack of reproducibility is clearly seen 
sults in a shrinkage of the scar tissue ("capsular contracture") and noticeable distortion of the reconstructed or augmented breast. PMRT increases the frequency and worsens the degree of capsular contracture, as tissue fibrosis is a well known late normal tissue effect of radiotherapy [19]. Severe capsular contracture, where revisional surgery in the form of capsulotomy or capsulectomy with implant exchange is usually required, is reported at the level of more than 30\% after IB-IBR and PMRT [4, 19]. Another major complications, i.e. requiring revisional surgery, are reported in one-third of patients after IB-IBR and PMRT [4].

Irradiated patients have an inferior cosmetic outcomes of IB-IBR: good or excellent cosmetic result attainable in about 90\% of patients with IB-IBR alone, decreases to 57\% after PMRT [20]. In two-stage prosthetic reconstruction, any sequence of PMRT (i.e. radiation to the $\mathrm{TE}$, or to the permanent implant) negatively impacts the final aesthetic outcome and long-term implant survival. In this setting, the risk of reconstructive failure is significantly higher for patients with PMRT to the TE compared to patients with PMRT to permanent implant (six-year predicted failure rates of $32 \%$ vs. $16.4 \%, p<0.01$ ), but the final aesthetic results and capsular contracture rates are slightly better [21].

The impact of PMRT on the cosmetic outcomes after prepectoral versus retropectoral IB-IBR has not been clearly defined to date. With the increasing rates of prepectoral reconstructions being performed, it is important to assess the outcomes in the setting of PMRT, to ensure that morbidity rates are not higher, as these patients undergo PMRT without the presence of vascularized muscle over the implants [22]. There is a growing body of evidence from retrospective data to suggest that prepectoral reconstruction is an effective technique in the setting of PMRT, with morbidity rates similar or even better than those experienced with complete submuscular or dual-plane (partial submuscular) coverage techniques with PMRT $[22,23]$. However, the use of ADM seems crucial for these patients, because it is believed that ADM may protect against capsular contracture after IB-IBR in the non-irradiated and PMRT settings, and the risk of extensive soft-tissue damage and expander exposure is greater in patients with prepectoral reconstruction without $\operatorname{ADM}[23,24]$.

In summary, patients should be appropriately counseled about all the aforementioned risks and consequences of potential complications so they could make fully informed decisions.

\section{Target volume definitions with respect to the reconstruction methods and disease stage}

Most of the local recurrences after mastectomy occur at the level of the skin and subcutaneous tissue (about 75\%) and within the pectoral muscle, especially near the primary tumour site (about 25\%) [25]. Thus, European Society for Radiotherapy and Oncology (ESTRO) consensus guidelines for target volume delineation in the setting of PMRT recommend that in case of retro-pectoral implants the CTV of the chest wall should be positioned ventral (anterior) to the major pectoral muscle [26].
In case of the retro-pectoral implants with partial coverage by the pectoral muscle and supportive material in the lower part, for patients with adverse factors or with the tumour localised close to the dorsal fascia, the ESTRO guidelines [26] recommend to include in the CTV the part of the chest wall that was initially not covered by the major pectoral muscle, taking into account the muscle's pre-surgical position (which preferably should be marked with surgical clips). After IBR using a prepectoral implant, the CTV is composed of 2 parts: the ventral part between the skin and the implant, containing the subcutaneous lymphatic plexus and eventual residual glandular tissue and the dorsal part between the implant and the pectoral muscle or the chest wall, containing eventual residual glandular tissue. This second part should be included in case of the presence of adverse tumour factors. In case of rib cage invasion, the ribs/intercostal muscles should also be included in the CTV, irrespectively of the reconstruction technique, however the guidelines emphasize that IBR is generally not advised in these patients. For selected patients with LABC considered for IBR, the CTV should be based on the discussion in a multidisciplinary team conference and carefully individually adapted per case, according to the high-risk areas for remaining subclinical tumour deposits. In case of ambiguities, it's recommended to include the entire mastectomy site including the implant [26]. Such a design of the CTV often results with less optimal dose distribution, and the risk for higher doses in normal tissues. The transplanted tissues (skin, fat, muscle) and synthetic materials (implant, TE, ADM) are not part of the CTV [26].

\section{Boost dose and the use of bolus}

According to the ESTRO guidelines [26], the use of a "tumour-bed" boost (i.e. additional radiotherapy dose) is not recommended, unless the surgeon has placed clips to mark anticipated and subsequently confirmed involved resection margins that cannot be removed surgically.

Bolus, i.e. the tissue equivalent material, is used in radiotherapy to provide build-up of dose to the skin surface. The main indication for the use of bolus after IB-IBR is skin involvement. As long as patients with skin involvement were not offered skin-sparing mastectomy, most of the European radiation oncologists did not use bolus [7], however nowadays the need for using bolus increases and up to two-thirds of radiation oncologists declare that they do not use bolus "unless the skin is involved" [10]. This may impact the aesthetic outcomes of IB-IBR, as the use of bolus was recognized as the only "technical" radiotherapy factor negatively influencing cosmetic results [27].

In patients with skin involvement who underwent IB-IBR, the use of bolus poses specific challenges, because to be able to fulfill its function, bolus material should adhere tightly to the skin. This is very often difficult or even impossible to achieve on the curved-shaped reconstructed breast (Fig. 2), resulting in the underdosage of the skin within the target volume, thus possibly influencing local control of the tumour. Offering the IB-IBR to the patients with skin involvement puts them at hi- 
gher risk of local relapse that might be avoided if the patient underwent mastectomy with breast reconstruction delayed after completion of oncological treatment.

\section{Tissue expander - deflated or inflated?}

The volume of fluid within the implant affects radiation dose distribution and can make radiation treatment planning challenging [7]. On the other hand - expansion of the TE after completion of PMRT is usually not possible due to radiation-induced early and late normal tissue effects. Thus, the approach to the patients for whom IB-IBR and PMRT follows the neoadjuvant chemotherapy involves rapid expansion of the TE within 6 weeks and start of radiation to the TE within 8 weeks post-surgery [8]. This usually means the moderate volume of fluid within the TE, as pushing the inflation to the maximal volume within this short period of time would mean very thin and tightened skin and subcutaneous tissues, more prone to radiation damage. In particular situations, radiation oncologists would ask reconstructive surgeons to adjust the TE volume to facilitate PMRT planning or to improve the predicted reproducibility of the target positioning, but it should be kept in mind that PMRT to the completely deflated TE could make it impossible to expand the TE in the future. In patients with bilateral IB-IBR with TEs, most of the radiation oncologists will request that the TE be deflated in the contralateral unaffected breast to minimize radiation dose to this breast [7, 28]. Of note, the volume of fluid within the TE (or both TEs in case of bilateral IB-IBR) has to remain the same during the whole course of

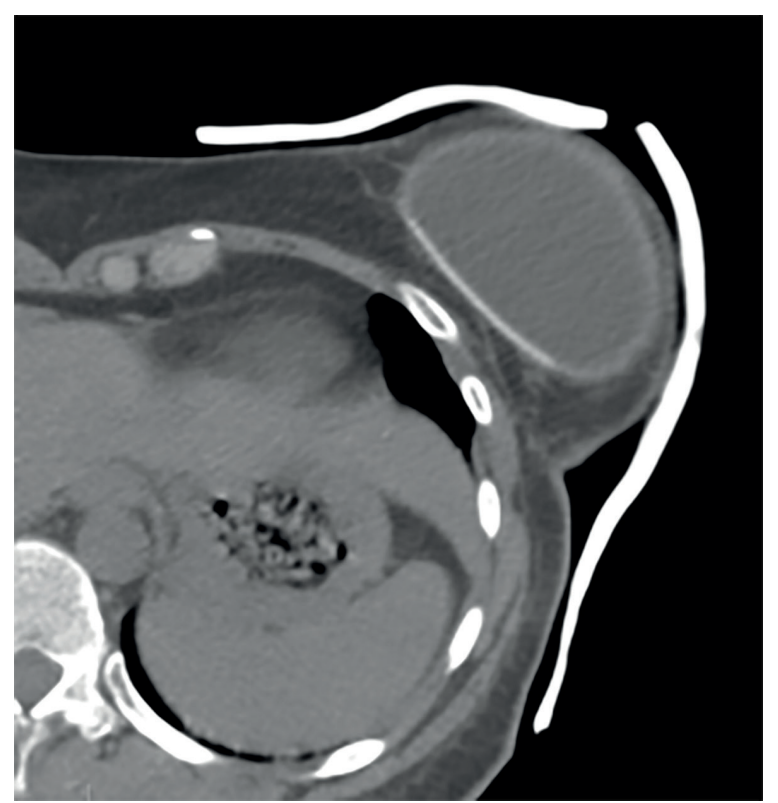

Figure 2. A bolus is a layer of tissue-equivalent material placed on the patient's skin during treatment that assists in providing the optimal dose of radiation. Bolus should adhere tightly to the skin, which is often difficult or even impossible on the curved-shaped reconstructed breast, resulting in underdosage of the skin within the target volume, thus possibly influencing local control of the tumour in patients with skin involvement
PMRT, starting on the day when the computed tomography for radiation treatment planning is performed.

The air-filled expanders (Fig. 3) are not suitable for irradiation, as the thin rim of tissues surrounded with air lies entirely within the build-up region (i.e. the layer between the surface and the depth of dose maximum; energy deposition increases gradually beneath the surface, reaching the equilibrium at a finite depth), so the dose distribution would be unacceptable, with significant underdosage of the CTV.

\section{Tissue expander - the impact of an internal magnetic metallic port}

Frequently used TEs contain the internal metallic ports with a strong magnet, through which the fluid is injected. The metallic port magnet is made of high-Z, high-density, rare-earth metal which results in artefacts in imaging and perturbation in dose distribution around the port when receiving PMRT to TE.

Dose is attenuated in the "shadow" of the TE port in patients receiving PMRT, with an average reduction of $7-13 \%$ in dose in vivo to skin surface, when compared with that predicted by the treatment planning system (TPS) [29-31]. This level of attenuation is considered likely to be clinically insignificant for most patients, but each centre should undertake its own appropriate measurements before utilizing TPS predictions [29].

Another dose perturbation is the increase in dose upstream of the metallic disk caused by backscatter and the dose beside the magnet caused by side scatter radiation. Backscatter measurements [30] showed that when the port is in the parallel orientation, i.e. parallel to the central axis of the beam, there is a $4 \%$ increase in dose close to the edge of the disk compared to the dose without the metallic disk, but this difference decreases rapidly farther from the disk edge and at distances greater than $3 \mathrm{~mm}$ there is no significant effect on

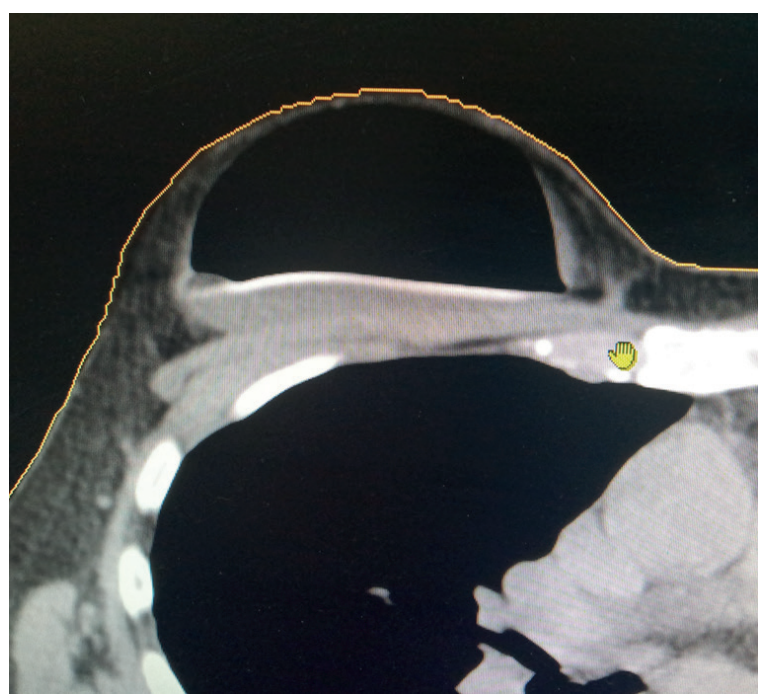

Figure 3. The air-filled expanders are not suitable for irradiation. The dose distribution would be unacceptable in the thin rim of tissues surrounded with air, with significant underdosage of the clinical target volume which in that case would be located within the build-up region 
the dose. This port setup is similar to the way the TE might be irradiated in a patient with a parallel-opposed pair tangential beam arrangement. When the port is perpendicular to the central axis of the beam (one of the possible positions of the port during an arc-therapy delivery), the increase in dose is larger: $11 \%$ at the disk edge, but again this decreases sharply away from the disk edge so that there is no effect on the dose beyond $5 \mathrm{~mm}$. Side scatter measurements [30] showed that there is an increase in dose of $2.75 \%$ compared to the dose without the metallic disk at the edge of the disk, decreasing to $0 \%$ at $7 \mathrm{~mm}$ away from the disk. Thus, the percentage increase in dose due to the scatter radiation is within the range acceptable in the treatment planning, and in case of the TE with an internal metallic port, the range of these secondary electrons scattered back from the implant is no more than $5 \mathrm{~mm}$ and should not result in an increase in dose to breast tissue, being absorbed in the silicone elastomer shell and saline components of the TE [30].

\section{Conclusion}

The complexity of integrating IB-IBR and PMRT underscores the need for close communication in multidisciplinary team to best prospectively coordinate and deliver patient-centered breast cancer care. Decision-making regarding the possibility of IB-IBR belongs to the surgeon and is based on the assessment of feasibility, the patient's characteristics and wishes, as well as the surgeon's skill and expertise, however - to offer breast cancer patients best outcomes in terms of disease control, toxicity, cosmesis and quality of life after reconstruction - surgeons and radiation oncologists need to develop "shared views" on risks and priorities for the particular patient. Thus, a radiation oncologist should always be present at the pre-surgery clinical meetings that plan breast reconstructions. Patients must be well informed, not only regarding potential benefits of IB-IBR, but also on the possibility of an increased risk of complications in the PMRT setting.

\section{Conflict of interest: none declared}

\section{Joanna Socha \\ Military Institute of Medicine \\ Department of Radiotherapy \\ ul. Szaserów 128 \\ 04-141 Warsaw, Poland \\ e-mail:sochajoanna@wp.pl}

\section{Received: 28 Jan 2020}

Accepted: 24 Feb 2020

\section{References}

1. McGale P, Taylor C, Correa C, et al. EBCTCG (Early Breast Cancer Trialists' Collaborative Group). Effect of radiotherapy after mastectomy and axillary surgery on 10-year recurrence and 20-year breast cancer mortality: meta-analysis of individual patient data for 8135 women in 22 randomised trials. Lancet. 2014; 383(9935): 2127-2135, doi: 10.1016/ S0140-6736(14)60488-8, indexed in Pubmed: 24656685.
2. Marks LB, Kaidar-Person O, Poortmans P. Regarding Current Recommendations for Postmastectomy Radiation Therapy in Patients With One to Three Positive Axillary Lymph Nodes. J Clin Oncol. 2017; 35(11): 1256 1258, doi: 10.1200/JCO.2016.71.0764, indexed in Pubmed: 28068174.

3. Frasier LL, Holden S, Holden T, et al.Temporal Trends in Postmastectomy Radiation Therapy and Breast Reconstruction Associated With Changes in National Comprehensive Cancer Network Guidelines. JAMA Oncol. 2016; 2(1): 95-101, doi: 10.1001/jamaoncol.2015.3717, indexed in Pubmed: 26539936.

4. Momoh AO, Ahmed R, Kelley BP, et al. A systematic review of complications of implant-based breast reconstruction with prereconstruction and postreconstruction radiotherapy. Ann Surg Oncol. 2014; 21 (1): 118 124, doi: 10.1245/s10434-013-3284-z, indexed in Pubmed: 24081801.

5. Kelley BP, Ahmed R, Kidwell KM, et al. A systematic review of morbidity associated with autologous breast reconstruction before and after exposure to radiotherapy: are current practices ideal? Ann Surg Oncol. 2014; 21(5): 1732-1738, doi: 10.1245/s10434-014-3494-z, indexed in Pubmed: 24473643.

6. Jagsi R, Jiang J, Momoh AO, et al. Complications After Mastectomy and Immediate Breast Reconstruction for Breast Cancer: A ClaimsBased Analysis. Ann Surg. 2016; 263(2): 219-227, doi: 10.1097/ SLA.0000000000001177, indexed in Pubmed: 25876011.

7. Chen SA, Hiley $C$, Nickleach D, et al. Breast reconstruction and post-mastectomy radiation practice. Radiat Oncol. 2013; 8: 45, doi: 10.1186/1748717X-8-45, indexed in Pubmed: 23452558.

8. Kuerer HM, Cordeiro PG, Mutter RW. Optimizing Breast Cancer Adjuvant Radiation and Integration of Breast and Reconstructive Surgery. Am Soc Clin Oncol Educ Book. 2017; 37: 93-105, doi: 10.1200/EDBK_175342, indexed in Pubmed: 28561684.

9. National Comprehensive Cancer Network, Breast Cancer, Version 1.2020. https://www.ncen.org/professionals/physician_gls/pdf/breast. pdf (24.01.2020).

10. Aristei C, Kaidar-Person O, Tagliaferri L, et al. The Assisi Think Tank Meeting and Survey of post MAstectomy Radiation Therapy after breast reconstruction: The ATTM-SMART report. Eur J Surg Oncol. 2018; 44(4): 436-443, doi: 10.1016/j.ejso.2018.01.010, indexed in Pubmed: 29422254.

11. Brennan ME, Flitcroft $\mathrm{K}$, Warrier $\mathrm{S}$, et al. Immediate expander/implant breast reconstruction followed by post-mastectomy radiotherapy for breast cancer: Aesthetic, surgical, satisfaction and quality of life outcomes in women with high-risk breast cancer. Breast. 2016; 30: 59-65, doi: 10.1016/j.breast.2016.08.008, indexed in Pubmed: 27611237.

12. Gieni M, Avram R, Dickson L, et al. Local breast cancer recurrence after mastectomy and immediate breast reconstruction for invasive cancer: a meta-analysis. Breast. 2012; 21(3): 230-236, doi: 10.1016/j. breast.2011.12.013, indexed in Pubmed: 22225710.

13. Zhang $\mathrm{P}, \mathrm{Li} \mathrm{CZ}, \mathrm{Wu} \mathrm{CT}$, et al. Comparison of immediate breast reconstruction after mastectomy and mastectomy alone for breast cancer: A meta-analysis. Eur J Surg Oncol. 2017; 43(2): 285-293, doi: 10.1016/j. ejso.2016.07.006, indexed in Pubmed: 27503441.

14. Bjöhle J, Onjukka E, Rintelä N, et al. Post-mastectomy radiation therapy with or without implant-based reconstruction is safe in terms of clinical target volume coverage and survival - A matched cohort study. Radiother Oncol. 2019; 131: 229-236, doi: 10.1016/j.radonc.2018.07.005, indexed in Pubmed: 30055939.

15. Wu SG, Zhang WW, Sun JY, et al. Comparison of survival outcomes of locally advanced breast cancer patients receiving post-mastectomy radiotherapy with and without immediate breast reconstruction: a population-based analysis. Cancer Manag Res. 2018; 10: 1993-2002, doi: 10.2147/CMAR.S162430, indexed in Pubmed: 30034254.

16. Haviland JS, Bentzen SM, Bliss JM, et al. START Trial Management Group. Prolongation of overall treatment time as a cause of treatment failure in early breast cancer: An analysis of the UK START (Standardisation of Breast Radiotherapy) trials of radiotherapy fractionation. Radiother Oncol. 2016; 121(3): 420-423, doi: 10.1016/j.radonc.2016.08.027, indexed in Pubmed: 27666929.

17. Chen TA, Momeni A, Lee GK. Clinical outcomes in breast cancer expander-implant reconstructive patients with radiation therapy. J Plast Reconstr Aesthet Surg. 2016; 69(1): 14-22, doi: 10.1016/j.bjps.2015.08.032, indexed in Pubmed: 26453182.

18. Lam TC, Hsieh F, Boyages J. The effects of postmastectomy adjuvant radiotherapy on immediate two-stage prosthetic breast reconstruction: a systematic review. Plast Reconstr Surg. 2013; 132(3): 511-518, doi: 10.1097/PRS.0b013e31829acc41, indexed in Pubmed: 23676964.

19. Whitfield GA, Horan G, Irwin MS, et al. Incidence of severe capsular contracture following implant-based immediate breast reconstruction with or without postoperative chest wall radiotherapy using 40 Gray 
in 15 fractions. Radiother Oncol. 2009; 90(1): 141-147, doi: 10.1016/j. radonc.2008.09.023, indexed in Pubmed: 18977547.

20. Lam TC, Borotkanics R, Hsieh F, et al. Immediate Two-Stage Prosthetic Breast Reconstruction Failure: Radiation Is Not the Only Culprit. Plast Reconstr Surg. 2018; 141(6): 1315-1324, doi: 10.1097/ PRS.0000000000004358, indexed in Pubmed: 29750759.

21. Cordeiro PG, Albornoz CR, McCormick B, et al. What Is the Optimum Timing of Postmastectomy Radiotherapy in Two-Stage Prosthetic Reconstruction: Radiation to the Tissue Expander or Permanent Implant? Plast Reconstr Surg. 2015; 135(6): 1509-1517, doi: 10.1097/ PRS.0000000000001278, indexed in Pubmed: 25742523.

22. Sbitany H, Gomez-Sanchez C, Piper M, et al. Prepectoral Breast Reconstruction in the Setting of Postmastectomy Radiation Therapy: An Assessment of Clinical Outcomes and Benefits. Plast Reconstr Surg. 2019; 143(1): 10-20, doi: 10.1097/PRS.0000000000005140, indexed in Pubmed: 30589770.

23. Sinnott CJ, Persing SM, Pronovost M, et al. Impact of Postmastectomy Radiation Therapy in Prepectoral Versus Subpectoral Implant-Based Breast Reconstruction. Ann Surg Oncol. 2018; 25(10): 2899-2908, doi: 10.1245/s10434-018-6602-7, indexed in Pubmed: 29978367.

24. Salibian AH, Harness JK, Mowlds DS. Staged Suprapectoral Expander/ Implant Reconstruction without Acellular Dermal Matrix following Nipple-Sparing Mastectomy. Plast Reconstr Surg. 2017; 139(1): 30-39, doi: 10.1097/PRS.0000000000002845, indexed in Pubmed: 28027223.

25. Vargo JA, Beriwal S. In reply to Chang et al.: Contouring guidelines for post-mastectomy radiotherapy a cry for international consensus. Radiother Oncol. 2017; 123(3): 483-484, doi: 10.1016/j.radonc.2017.05.005, indexed in Pubmed: 28528018.
26. Kaidar-Person O, Vrou Offersen $B$, Hol S, et al. ESTRO ACROP consensus guideline for target volume delineation in the setting of postmastectomy radiation therapy after implant-based immediate reconstruction for early stage breast cancer. Radiother Oncol. 2019; 137: 159-166, doi: 10.1016/j.radonc.2019.04.010, indexed in Pubmed: 31108277.

27. Kuske RR, Schuster R, Klein E, et al. Radiotherapy and breast reconstruction: clinical results and dosimetry. Int J Radiat Oncol Biol Phys. 1991; 21(2): 339-346, doi: 10.1016/0360-3016(91)90780-8, indexed in Pubmed: 2061110.

28. Thomas K, Rahimi A, Spangler A, et al. Radiation practice patterns among United States radiation oncologists for postmastectomy breast reconstruction and oncoplastic breast reduction. Pract Radiat Oncol. 2014; 4(6): 466-471, doi: 10.1016/j.prro.2014.04.002, indexed in Pubmed: 25407870.

29. Gee HE, Bignell F, Odgers $D$, et al. In vivo dosimetric impact of breast tissue expanders on post-mastectomy radiotherapy. J Med Imaging Radiat Oncol. 2016; 60(1): 138-145, doi: 10.1111/1754-9485.12403, indexed in Pubmed: 26503758.

30. Thompson RCA, Morgan AM. Investigation into dosimetric effect of a MAGNA-SITE tissue expander on post-mastectomy radiotherapy. Med Phys. 2005; 32(6): 1640-1646, doi: 10.1118/1.1914545, indexed in Pubmed: 16013723.

31. Chatzigiannis C, Lymperopoulou G, Sandilos $P$, et al. Dose perturbation in the radiotherapy of breast cancer patients implanted with the Magna-Site: a Monte Carlo study. J Appl Clin Med Phys. 2011; 12(2): 3295, doi: 10.1120/jacmp.v12i2.3295, indexed in Pubmed: 21587170. 